\title{
Tournaments as collective decisions
}

Citation for published version (APA):

Storcken, T. (2021). Tournaments as collective decisions. Maastricht University, Graduate School of Business and Economics. GSBE Research Memoranda No. 015 https://doi.org/10.26481/umagsb.2021015

Document status and date:

Published: 30/09/2021

DOI:

10.26481/umagsb.2021015

Document Version:

Publisher's PDF, also known as Version of record

\section{Please check the document version of this publication:}

- A submitted manuscript is the version of the article upon submission and before peer-review. There can be important differences between the submitted version and the official published version of record.

People interested in the research are advised to contact the author for the final version of the publication, or visit the DOI to the publisher's website.

- The final author version and the galley proof are versions of the publication after peer review.

- The final published version features the final layout of the paper including the volume, issue and page numbers.

Link to publication

\footnotetext{
General rights rights.

- You may freely distribute the URL identifying the publication in the public portal. please follow below link for the End User Agreement:

www.umlib.nl/taverne-license

Take down policy

If you believe that this document breaches copyright please contact us at:

repository@maastrichtuniversity.nl

providing details and we will investigate your claim.
}

Copyright and moral rights for the publications made accessible in the public portal are retained by the authors and/or other copyright owners and it is a condition of accessing publications that users recognise and abide by the legal requirements associated with these

- Users may download and print one copy of any publication from the public portal for the purpose of private study or research.

- You may not further distribute the material or use it for any profit-making activity or commercial gain

If the publication is distributed under the terms of Article $25 \mathrm{fa}$ of the Dutch Copyright Act, indicated by the "Taverne" license above, 


\section{Maastricht University}

Ton Storcken

Tournaments as collective decisions

$\mathrm{RM} / 21 / 015$

ISSN: $2666-8807$

\section{GSBE}

Maastricht University School of Business and Economics

Graduate School of Business and Economics

P.O Box 616

NL-6200 MD Maastricht

The Netherlands 


\title{
Tournaments as collective decisions
}

\author{
Ton Storcken, Maastricht University
}

September 2021

\begin{abstract}
Unless all tournaments are admissible as individual preferences, we show that, structure diversity of the range of a Pareto-optimal, neutral, non-dictatorial, and independent of irrelevant alternatives preference rules is greater than the structure diversity in the individual preferences upon which these preference rules are based.
\end{abstract}

\section{Introduction}

The works of e.g. Paul Anand (1993) and Amos Tversky (1969) show individual preferences may be cyclic. It is therefore too restrictive to impose that collective decisions are transitive or acyclic. Apart from the problem of finding a "best" or "maximal" alternative when the outcome of a collective preference exhibits cycles, this paper demonstrates that the fundamental impossibilities for collective decision making, as spelled out in Kenneth Arrow (1978), translates to such cyclical situations and therewith reveals even deeper insight.

To be more explicit, allowing for cyclical outcome preferences at collective decisions allows for instance for the pairwise majority rule. A result of this paper is that the "structure" diversity of the individual preferences is less than that of the collective preferences. Applying the pairwise majority rule yields "structures" in the outcome preferences which are not present at the individual preferences. This paper shows that this holds for all preferences rules satisfying Pareto optimality, neutrality, the independence of irrelevant alternatives, and non-dictatorship. So, applying this result to the classical Arrow framework of for instance linear orders, Theorem 1 means that a preference rule satisfying Pareto optimal, neutrality, and the independence of irrelevant alternatives, is either dictatorial or its range has to allow for cyclical structural components. As the latter is excluded by the (classical) model, it follows that such a preference rule is dictatorial. In many impossibility theorems the neutrality condition is not imposed. Lemma 3 shows that under mild transitivity assumptions, which capture all well-known types of transitivity, neutrality is implied by the other conditions. This explains the absence of neutrality in many well-known impossibility theorems.

In case cyclical structures are admissible in individual and collective preferences the regular transitivity or acyclicity conditions used to describe a domain 
of preferences cannot be used. Here, like Harrie de Swart ea. (1992) we specify a domain of individual preferences by a set of tournaments that is closed under some operations. These operations are permutation, reversion, and cut and paste. Closed under permutation means that the naming the alternatives is immaterial to being a preference in the domain. If closedness under permutations is not satisfied, then a condition like neutrality cannot be defined. Closed under reversion means that reversing all pairs in a preference yield a new preference which is in the domain. Seeing a preference like putting alternatives in a "left-right", "big-small", "young-old", etc. frame work the choice of where to assign "left", "big", "young" etc. is arbitrary. So, the reversed preference is also a preference. Closed under cut and paste means that ordering disjoint parts of various preferences strictly and therewith obtaining a tournament yields a preferences. Roughly speaking, being closed under cut and paste means that preferences are described by their parts.

To the best of our knowledge, there is no literature on cyclical individual collective preferences yielding impossibility results like Theorems 1 stating that the range of a Pareto optimal, neutral, independent of irrelevant alternatives and non-dictatorial preference rule is more "structure" diverse than its domain or like Theorem 4 which spells out a lower bound for this diversity. There are many impossibility theorems of this kind where the domain and the range satisfy certain transitivity or a-cyclicity conditions. Theorem 2 relates our results to many of these, in particular, those concerning complete preferences. The purpose of this paper is not to create a unifying theory of all these impossibility results. We therefore refrain from an elaborate comparison of the results presented here with those in literature meant above. This because such a comparison would distract attention from the theoretical account presented here that collective decision making is more difficult than individual decision making as the collective outcome preferences may embody more structure diversity than the individual preferences.

The paper is structured as follows. Section 2 describes the model. Section 3 discusses why structural diversity increases when going from individual to collective preferences. Section 4 shows that this result relates to the existing impossibility results. And, Section 5 strengthens the result Section 3 in determining some lower bounds on this diversity.

\section{Tournaments as preferences}

For a finite set $S$ let $\# S$ denote its cardinality. Let $N=\{1,2,3, \ldots, n\}$ denote the finite set of $n$ agents. Agents are usually indicated by variables $i$ and $j$. Let $A$ denote a finite set of $m$ alternatives. Alternative are usually denoted by variables $a, b, c, x, y$, and $z$. Let $\mathbb{T}$ denote the set of all tournaments on $A$. These are binary relations on $A$ which are complete, therewith reflexive, and anti-symmetric.

Let $R$ and $R^{\prime}$ be tournaments in $\mathbb{T}$. It is standard to interpret $(a, b) \in R$ as $a$ is (strictly) ordered above $b$ at $R$. Let $\sigma$ be a permutation of $A$. The permutation 
of $R$ is defined by $\sigma R=\{(\sigma(x), \sigma(y)):(x, y) \in R\}$. The reversed of $R$ is defined by $-R=\{(b, a):(a, b) \in R\}$. Let $B$ be an non-empty subset of $A$. The restriction of $R$ to $B$ is defined by $\left.R\right|_{B}=(R \cap(B \times B))$. Note that if $B$ equals $A$, then $R=\left.R\right|_{B}$. For disjoint non-empty subsets $B_{1}$ and $B_{2}$ of $B$ the concatenation of $\left.R\right|_{B_{1}}$ and $\left.R^{\prime}\right|_{B_{2}}$ is defined by $\left.\left.R\right|_{B_{1}} \gg R^{\prime}\right|_{B_{2}}=\left.\left.R\right|_{B_{1}} \cup\left(B_{1} \times B_{2}\right) \cup R^{\prime}\right|_{B_{2}}$. So, at $\left.\left.R\right|_{B_{1}} \gg R^{\prime}\right|_{B_{2}}$ all alternatives in $B_{1}$ are ordered like in $R$, all alternatives in $B_{2}$ are ordered like in $R^{\prime}$ and all alternatives in $B_{1}$ are ordered above all alternatives in $B_{2}$. It is straightforward to see that concatenation is associative. A (sub)tournament $\left.R\right|_{B}$ is called reducible if there are non-empty subset $B_{1}$ and $B_{2}$ of $B$ such that $\left.R\right|_{B}=\left.\left.R\right|_{B_{1}} \gg R\right|_{B_{2}}$. We call $\left.R\right|_{B}$ irreducible, it is not reducible.

Note that linear orders are transitive tournaments. The set of linear orders, denoted by $\mathbb{L}$, is a subset of the set of tournaments. In many studies on Social Choice Theory the set of individual admissible preferences is equal to $\mathbb{L}$. Here the set of admissible preferences, denoted by $\mathbb{D}$, is taken more general. We assume that $\mathbb{D}$ is a domain of preferences, meaning that it is a non-empty subset of $\mathbb{T}$ that is closed under

1. permutations, i.e. for all permutations $\sigma$ of $A$ and all tournaments $R$ in $\mathbb{D}$ the permuted tournament $\sigma R$ is in $\mathbb{D}$,

2. reversion, i.e. for all tournaments $R$ in $\mathbb{D}$ the reversed tournament $-R$ is in $\mathbb{D}$,

3. cut and paste, i.e. for all non-trivial subsets $B$ of $A$ and all tournaments $R$ and $R^{\prime}$ in $\mathbb{D}$ tournament $\left.\left.R\right|_{B} \gg R^{\prime}\right|_{(A \backslash B)}$ is in $\mathbb{D}$.

Being closed under permutations for an domain of admissible preferences means that the roles/positions of alternatives in the admissible preferences can be swapped. This prevents discrimination between alternatives. Being closed under reversion prevents discrimination of the "direction" to which we order. Orders on a scale from left to right, from high to low, from good to bad, from best to worst, etc... are reversible yielding an order on a scale from right to left, from low to high, from bad to good, from worst to best, respectively. Here concatenation is considered to be a basic idea of ordering as it puts parts of tournaments in line: left to right or best to worst. In view of this cut and past means that parts of admissible preferences are admissible.

It is straightforward to check that $\mathbb{L}$ is closed under permutations, reversion, and under cut and paste. Further, from any tournament by a sequence of cut and paste operations we may construct any given linear order. This means that $\mathbb{L}$ is a subset of any domain of preferences. Also we have the following basic observation that being closed under permutations, reversion and cut and past is intersection robust. 
Proposition 1 Let $\mathbb{U}$ and $\mathbb{V}$ be two sets of tournaments.

1. If $\mathbb{U}$ and $\mathbb{V}$ are both closed under permutations, then $\mathbb{U} \cap \mathbb{V}$ is closed under permutations,

2. If $\mathbb{U}$ and $\mathbb{V}$ are both closed under reversions, then $\mathbb{U} \cap \mathbb{V}$ is closed under reversion, and

3. If $\mathbb{U}$ and $\mathbb{V}$ are both closed under cut and paste, then $\mathbb{U} \cap \mathbb{V}$ is closed under cut and paste.

Proof. To proof the implication at (1) let both $\mathbb{U}$ and $\mathbb{V}$ be closed under permutations. Let $R \in \mathbb{U} \cap \mathbb{V}$ and let $\sigma$ be a permutation of $A$. It is sufficient to prove that $\sigma R \in \mathbb{U} \cap \mathbb{V}$. As $R \in \mathbb{U} \cap \mathbb{V}, R \in \mathbb{U}$. As $\mathbb{U}$ is closed under permutations it follows that $\sigma R \in \mathbb{U}$. Similarly it follows that $\sigma R \in \mathbb{V}$. So, $\sigma R \in \mathbb{U} \cap \mathbb{V}$.

To proof the implication at (2) let both $\mathbb{U}$ and $\mathbb{V}$ be closed under reversion. Let $R \in \mathbb{U} \cap \mathbb{V}$. It is sufficient to prove that $-R \in \mathbb{U} \cap \mathbb{V}$. As $R \in \mathbb{U} \cap \mathbb{V}, R \in \mathbb{U}$. As $\mathbb{U}$ is closed under reversion it follows that $-R \in \mathbb{U}$. Similarly it follows that $-R \in \mathbb{V}$. So, $-R \in \mathbb{U} \cap \mathbb{V}$.

To proof the implication at (3) let both $\mathbb{U}$ and $\mathbb{V}$ be closed under cut and paste. Let $R$ and $R^{\prime}$ be in $\mathbb{U} \cap \mathbb{V}$ and let $B$ be a non-trivial subset of $A$. It is sufficient to prove that $\left.\left.R\right|_{B} \gg R^{\prime}\right|_{(A \backslash B)} \in \mathbb{U} \cap \mathbb{V}$. As $R$ and $R^{\prime}$ are in $\mathbb{U} \cap \mathbb{V}, R$ and $R^{\prime}$ are in $\mathbb{U}$. As $\mathbb{U}$ is closed under cut and paste it follows that $\left.\left.R\right|_{B} \gg R^{\prime}\right|_{(A \backslash B)} \in \mathbb{U}$. Similarly it follows that $\left.\left.R\right|_{B} \gg R^{\prime}\right|_{(A \backslash B)} \in \mathbb{V}$. So, $\left.\left.R\right|_{B} \gg R^{\prime}\right|_{(A \backslash B)} \in \mathbb{U} \cap \mathbb{V}$.

Let $\mathbb{S}$ be an arbitrary set of tournaments. By Proposition 1 we can find the smallest domain of preferences containing $\mathbb{S}$ as follows

$$
\Delta(\mathbb{S})=\cap\{\mathbb{D}: \mathbb{S} \subset \mathbb{D} \text { and } \mathbb{D} \text { is a domain of preferences }\} .
$$

It is clear that many sets of tournaments are satisfying these three closedness conditions. Let $v$ and $w$ be positive integers. Let $\mathbb{T}_{v, w}$ denote the set of those tournaments $T$ for which there are a number, say $k$, a partition $B^{1}, B^{2}, \ldots, B^{k}$ of $A$ and sub-tournaments $\left.T^{1}\right|_{B^{1}},\left.T^{2}\right|_{B^{2}}, \ldots$, and $\left.T^{k}\right|_{B^{k}}$, with

1. $\# B^{t} \leq v$,

2. \#(T $\left.\left.\left.T^{t}\right|_{B^{t}} \backslash R^{t}\right|_{B^{t}}\right) \leq w$ for some $R^{t} \in \mathbb{L}$, and

3. $T=\left.\left.T^{1}\right|_{B^{1}} \gg T^{2}\right|_{B^{2}} \gg \ldots .\left.\gg T^{k}\right|_{B^{k}}$.

As $\# B^{t} \leq v$, the cycle length in tournaments in $\mathbb{T}_{v, w}$ is at most $v$ and irreducible parts $\left.T^{t}\right|_{B^{t}}$ are at most $w$ preference swaps away from a linear order. Sets $\mathbb{T}_{v, w}$ are examples of domains of preferences. Although this is straightforward to check we may also refer to Harrie de Swart ea. (1992).

Let $I$ denote the identity relation on $A$, i.e. $I=\{(x, x): x \in A\}$. Restrictions, permutations and cut and paste operations may be applied to $I$ yielding the obvious results. 
Let $\mathbb{D}^{N}$ denote the set of profiles. A profile $p$ is a function from $N$ to $\mathbb{D}$. Tournament $p(i)$ denotes the preference of agent $i$ at profile $p$. Permutations, restrictions, and cut and paste operations on tournaments can be extended to profiles component-wise as follows. Let $p$ and $q$ be two profiles in $\mathbb{D}^{N}$. Let $\sigma$ be a permutation on $A$. Let $B$ be a non-trivial subset of $A$.Then, $\sigma p$ is the permuted profile such that for all agents $i$ in $N(\sigma p)(i)=\sigma(p(i))$. Further, $\left.p\right|_{B}$ is the restricted profile such that for all agents $i$ in $N\left(\left.p\right|_{B}\right)(i)=\left(\left.p(i)\right|_{B}\right)$. The reversed profile $-p$ of $p$ is defined by $(-p)(i)=-p(i)$ for all agents $i$ in $N$. And $\left.\left.p\right|_{B} \gg q\right|_{(A \backslash B)}$ is the cut and past result defined coordinate-wise by $\left(\left.\left.p\right|_{B} \gg q\right|_{(A \backslash B)}\right)(i)=\left.\left.p(i)\right|_{B} \gg q(i)\right|_{(A \backslash B)}$ for all agents $i$ in $N$. Note that because $\mathbb{D}$ is closed under permutations and under cut and paste the coordinatewise extensions defined above are in $\mathbb{D}^{N}$.

In this paper collective decisions are formalized by preference rules, i.e. a function $\varphi$ from $\mathbb{D}^{N}$ to $\mathbb{T}$. We say that a coalition $S$, i.e. a subset of $N$, is winning if for all distinct alternatives $a$ and $b$ and profiles $p$ such that $(a, b) \in$ $\cap\{p(i): i \in S\}$ and $(b, a) \in \cap\{p(i): i \in N \backslash S\}$

$$
(a, b) \in \varphi(p) \text {. }
$$

So, $S$ is winning if at every profile $p$ the collective preference $\varphi(p)$ entails all those pairs where $S$ unanimously agrees upon and $N \backslash S$ unanimously opposes upon. We consider the following four conditions for preference rules

- Pareto optimality for all profiles $p$

$$
\cap\{p(i): i \in N\} \subseteq \varphi(p),
$$

- Neutrality for all profiles $p$ and all permutations $\sigma$ of $A$

$$
\varphi(\sigma p)=\sigma(\varphi(p))
$$

- Independence of irrelevant alternatives for all profiles $p$ and $q$ and all alternatives $a$ and $b$

$$
\left.p\right|_{\{a, b\}}=\left.q\right|_{\{a, b\}} \text { implies }\left.\varphi(p)\right|_{\{a, b\}}=\left.\varphi(q)\right|_{\{a, b\}},
$$

- No winning single agents for all agents $i$ in $N$

$\{i\}$ is not winning.

A simple game is a pair $(N, W)$, where $N$ is the set of agents and $W$ a set of subsets of $N$. We assume that $N$ is in $W$. Sets $S$ in $W$ are called winning. Simple game $(N, W)$ is called

- proper if $N \backslash S \notin W$ for all $S \in W$,

- strong if $N \backslash S \in W$ for all $S \subset N$ and $S \notin W$,and 
- zero one if $\{i\} \notin W$ for all $i \in N$.

\section{Remark 1 Simple Game}

Consider a neutral preference rules $\varphi$ that is independent of irrelevant alternatives. Then for all $S \subseteq N$

$$
\begin{aligned}
& S \text { is winning } \\
& \text { if and only if } \\
& \text { for some distinct alternatives } x \text { and } y \text { and some profile } p \text { in } \mathbb{D}^{N} \\
(x, y) \in & \varphi(p),(x, y) \in \cap\{p(i): i \in S\} \text { and }(y, x) \in \cap\{p(i): i \in N \backslash S\} .
\end{aligned}
$$

Let $\varphi$ be a Pareto optimal and neutral preference rule that is independent of irrelevant alternatives. Let $\varphi$ have no winning single agents. By the foregoing equivalence we can associate to $\varphi$ a unique simple game $\mathcal{G}^{\varphi}=\left(N, W^{\varphi}\right)$, where $W^{\varphi}$ consists of those subsets of $N$ that are winning at $\varphi$. Because $\varphi$ is neutral and independent of irrelevant alternatives and assigns a complete and antisymmetric relation to any profile $p$ we have that this simple game is proper and strong. As $\varphi$ has no winning single agents this simple game is zero one.

Reversely we can associate to each zero one, proper and strong simple game $\mathcal{G}=(N, W)$ a neutral and Pareto optimal preference rule $\varphi^{\mathcal{G}}$ that has no single winning agents and that is independent of irrelevant alternatives, where at an arbitrary profile $p$ in $\mathbb{D}^{N}$ outcome $\varphi^{\mathcal{G}}(p)$ contains all those pairs of alternatives $(a, b)$, such that

$$
a=b \text { or }\{i \in N:(a, b) \in p(i)\} \in W .
$$

It is straightforward to see that $\varphi^{\mathcal{G}^{\varphi}}=\varphi$ and $\mathcal{G}^{\varphi^{\mathcal{G}}}=\mathcal{G}$ for appropriate preference rules $\varphi$ and simple games $\mathcal{G}$.

Example 1 Odd preference rule

Preference rule $\varphi^{\text {odd }}$ satisfies Pareto optimality, neutrality and the independence of irrelevant alternatives. Although it is not dictatorial in the usual sense agents 1, 2 and 3 are all three winning. It is defined for an arbitrary profile $p$ and arbitrary tuple of alternatives $a$ and $b$ as follows

$$
(a, b) \in \varphi^{o d d}(p) \text { if } \#\{i \in\{1,2,3\}:(a, b) \in p(i)\} \text { is odd. }
$$

Lemma 1 Let preference rule $\varphi$ be Pareto optimal, neutral and independent of irrelevant alternatives. Further, assume that there are no winning agents at $\varphi$. Then there are three winning coalitions, say $S, T$, and $V$, such that $S \cap T, S \cap V$, and $T \cap V$ partition $N$.

Proof. Consider a winning coalition $S$ such that each of its subsets is not winning. Because $\mathcal{G}^{\varphi}$ is a zero one and proper simple game we have that $n>$ $\# S \geq 2$. Take $i \in S$. Then by the choice of $S$ the coalition $S \backslash\{i\}$ is not winning. But then as $\mathcal{G}^{\varphi}$ is a strong simple game $T=N \backslash(S \backslash\{i\})$ is winning. Take $V=N \backslash\{i\}$. As $\{i\}$ is not winning at $\varphi$ and $\mathcal{G}^{\varphi}$ is a strong simple game $N \backslash\{i\}$ is winning. Note that, $S \cap T=\{i\}, S \cap V=S \backslash\{i\}$, and $T \cap V=N \backslash S$. This completes the proof. 


\section{The structure diversity difference between in- dividual and collective preferences}

Consider linear order $\widehat{R}=\left\{\left(a_{s}, a_{t}\right): 1 \leq s \leq t \leq m\right\}$ ordering $a_{1}$ best, $a_{2}$ second best, $a_{3}$ third best and so on to $a_{m}$ which is ordered worst. It follows straightforwardly that $\mathbb{L}=\Delta(\{\widehat{R}\})$. So, the set of linear orders is the smallest domain of preferences containing linear order $\widehat{R}$.

Next, consider tournament $\widehat{T}_{3}=\left(\widehat{R} \backslash\left\{\left(a_{1}, a_{3}\right)\right\}\right) \cup\left\{\left(a_{3}, a_{1}\right)\right\}$ having a 3 -cycle, that is a cycle of length three, at the top and for the rest coincides with $\widehat{R}$. Using cut and paste any linear order can be obtained from $\widehat{T}_{3}$. Permutations may map the cycle on every triple of alternatives. Noting that every 3 -cycle is only one preference swap away from a linear order, it is elementary, to verify that $\Delta\left(\left\{\widehat{T}_{3}\right\}\right)=\mathbb{T}_{3,1}$. So, $\mathbb{T}_{3,1}$ is the smallest domain of preferences containing a tournament with precisely one top 3-cycle. Taking $\widehat{T}_{k}=\left(\widehat{R} \backslash\left\{\left(a_{1}, a_{k}\right)\right\}\right) \cup$ $\left\{\left(a_{k}, a_{1}\right)\right\}$, for $k \geq 3$, a tournament with precisely one top cycle of length $k$ being precisely one preference swap away from a linear order. It is elementary to deduce that $\Delta\left(\left\{\widehat{T}_{k}\right\}\right)=\mathbb{T}_{k, 1}$. So, $\mathbb{T}_{k, 1}$ is the smallest domain of preferences containing a tournament with precisely one top cycle of length $k$ that is precisely one preference swap away from a linear order.

The previous shows that many subsets of the set of tournaments are domains of preferences. These differ from each other by the "structures" which are admissible in these preferences. For instance, the examples above allow for structures obtained from a linear order in which only the pair "(best, worst)" is reversed to "(worst,best)". Like in Storcken and de Swart(1992) differences between domains of preferences are based on such "basic structures" as preferences result from concatenations of these. For example preferences in $\mathbb{T}_{3,1}$ may have cycles of length 3 , a basic structure which is not present in linear orders. On the other hand, preferences in $\mathbb{T}_{3,1}$ do not have cycles of length 4 . This means that a path of length 3 , going along 4 distinct alternatives has a direct short cut from its start to its end. Regularly, together with completeness and strictness conditions, such "transitivity conditions" are used to distinguish different types of preferences. Here these transitivity conditions do not work for instance to clarify the difference between $\mathbb{T}_{5,2}$ and $\mathbb{T}_{5,3}$. As preferences may be cyclical, more "subtle" distinctions between preferences are needed to enable the expression of our results.

In different domains of preferences different "basic structures" may be ordered "linearly", i.e. one after an other. Thereby, for arbitrary situations it is not clear whether based ion these "basic structures" a sensible comparison exists. However, if one domain, say $\mathbb{D}_{1}$, is a strict subset of the other, say $\mathbb{D}_{2}$, i.e. $\mathbb{D}_{1} \varsubsetneqq \mathbb{D}_{2}$, then we call the latter more structure diverse. Indeed in that case all "basic structure" present among some preferences in $\mathbb{D}_{1}$ are, via this presumed inclusion, also present in some of the preferences in $\mathbb{D}_{2}$. But as this presumed inclusion is strict some "structures" found among the preferences in $\mathbb{D}_{2}$ cannot be found among those in $\mathbb{D}_{1}$, as otherwise by the closedness conditions the domains of preferences would be equal. 
Next, we will compare the structure diversity of a domain of preferences $\mathbb{D}$ with the structure diversity of the range $\varphi\left(\mathbb{D}^{N}\right)$ of a neutral and Pareto optimal preference rule $\varphi$ that is independent of irrelevant alternatives. The following Proposition shows that this range $\varphi\left(\mathbb{D}^{N}\right)$ is a domain of preferences.

Proposition 2 Let $\mathbb{D}$ be a domain of preferences. Let $\varphi$ be a neutral and Pareto optimal preference rule that is independent of irrelevant alternatives on $\mathbb{D}^{N}$. Then $\varphi\left(\mathbb{D}^{N}\right)$ the range of $\varphi$ is a domain of preferences. Furthermore, $\mathbb{D} \subseteq$ $\varphi\left(\mathbb{D}^{N}\right)$.

Proof. Let $R, R^{\prime} \in \varphi\left(\mathbb{D}^{N}\right)$. Let $\emptyset \neq B \varsubsetneqq A$ and let $\sigma$ be a permutation of $A$. In order to prove that the range $\varphi\left(\mathbb{D}^{N}\right)$ of $\varphi$ is closed under permutations, reversion, and cut and paste it is sufficient to show that $\sigma R \in \varphi\left(\mathbb{D}^{N}\right),-R \in \varphi\left(\mathbb{D}^{N}\right)$, and $\left.\left.R\right|_{B} \gg R^{\prime}\right|_{(A \backslash B)} \in \varphi\left(\mathbb{D}^{N}\right)$. As $R, R^{\prime} \in \varphi\left(\mathbb{D}^{N}\right)$ there are profiles $p$ and $p^{\prime}$ in $\mathbb{D}^{N}$ such that $\varphi(p)=R$ and $\varphi\left(p^{\prime}\right)=R^{\prime}$.

Neutrality now implies that $\varphi(\sigma p)=\sigma \varphi(p)=\sigma R$. So, $\sigma R \in \varphi\left(\mathbb{D}^{N}\right)$.

To show that $-R \in \varphi\left(\mathbb{D}^{N}\right)$ let $(a, b) \in R$ for some distinct alternatives $a$ and $b$. It is sufficient to show that $(b, a) \in \varphi(-p)$. Let $\tau$ be the permutation of $A$ such that $\tau(a)=b, \tau(b)=a$ and $\tau(x)=x$ for all alternatives $x \in A \backslash\{a, b\}$. Note that $-\left.p\right|_{\{a, b\}}=\left.\tau p\right|_{\{a, b\}}$. So, by the independence of irrelevant alternatives we have $\left.\varphi(-p)\right|_{\{a, b\}}=\left.\varphi(\tau p)\right|_{\{a, b\}}$. As $(a, b) \in R=\varphi(p)$ neutrality implies $(b, a)=(\tau(a), \tau(b)) \in \varphi(\tau p)$. So, $(b, a) \in \varphi(-p)$.

Take $q=\left.\left.p\right|_{B} \gg p^{\prime}\right|_{(A \backslash B)}$. Because $\mathbb{D}$ is closed with respect to cut and paste $q$ is in $\mathbb{D}^{N}$. As $\left.p\right|_{B}=\left.q\right|_{B}$ and $\left.p^{\prime}\right|_{(A \backslash B)}=\left.q\right|_{(A \backslash B)}$ the independence of irrelevant alternative implies $\left.\varphi(q)\right|_{B}=\left.\varphi(p)\right|_{B}=\left.R\right|_{B}$ and $\left.\varphi(q)\right|_{(A \backslash B)}=$ $\left.\varphi\left(p^{\prime}\right)\right|_{(A \backslash B)}=\left.R^{\prime}\right|_{(A \backslash B)}$. Pareto optimality implies that $B \times(A \backslash B) \subset \varphi(q)$. So, $\left.\left.R\right|_{B} \gg R^{\prime}\right|_{(A \backslash B)}=\varphi(q) \in \varphi\left(\mathbb{D}^{N}\right)$.

To prove the furthermore part let $R \in \mathbb{D}$. It is sufficient to prove $R \in \varphi\left(\mathbb{D}^{N}\right)$. Consider unanimous profile $r$ in $\mathbb{D}^{N}$ such that $r(i)=R$ for all agents $i$. Pareto optimality implies that $\varphi(r)=R$.So, $R \in \varphi\left(\mathbb{D}^{N}\right)$.

Theorem 1 shows that the range of a Pareto optimal and neutral preference rule which is independent of irrelevant alternatives and which has no single winning agents is more structure diverse than that of the domain unless the domain consists of all possible tournaments. Lemma 2 prepares the proof of this Theorem.

Lemma 2 Let $\mathbb{D}$ be a domain of preferences. Let $R \in \mathbb{T}$ and $R \notin \mathbb{D}$. Then for some subset of alternatives $X=\left\{x_{1}, x_{2}, \ldots, x_{k}\right\}$,

1. $\left.\left.\left.R\right|_{(A \backslash X)} \gg I\right|_{\left\{x_{1}\right\}} \gg \ldots \gg I\right|_{\left\{x_{k}\right\}} \notin \mathbb{D}$ and

2. $\left.\left.\left.\left.R\right|_{(A \backslash(X \cup\{y\}))} \gg I\right|_{\left\{x_{1}\right\}} \gg \ldots \gg I\right|_{\left\{x_{k}\right\}} \gg I\right|_{\{y\}} \in \mathbb{D}$ for all $y \in A \backslash X$.

Furthermore, if $\#\left(R \backslash R^{\prime}\right)=1$ for some $R^{\prime}$ in $\mathbb{D}$, \#(R| $\left.\left.\right|_{(A \backslash X)} \gg I\right|_{\left\{x_{1}\right\}} \gg$ $\left.\left.\left.\left.\left.\ldots \gg I\right|_{\left\{x_{k}\right\}} \backslash R^{\prime}\right|_{(A \backslash X)} \gg I\right|_{\left\{x_{1}\right\}} \gg \ldots \gg I\right|_{\left\{x_{k}\right\}}\right)=1$.

Proof. Let $X=\left\{x_{1}, x_{2}, \ldots, x_{k}\right\}$. We may choose subset $X$ of $A$ such that $\# X=k$ is maximal and $R=\left.\left.\left.R\right|_{(A \backslash X)} \gg I\right|_{\left\{x_{1}\right\}} \gg \ldots \gg I\right|_{\left\{x_{k}\right\}}$ is not in $\mathbb{D}$. 
Because \#X is maximal we have $\left.\left.\left.\left.R\right|_{(A \backslash(X \cup\{y\}))} \gg I\right|_{\{y\}} \gg I\right|_{\left\{x_{1}\right\}} \gg \ldots \gg I\right|_{\left\{x_{k}\right\}}$ is in $\mathbb{D}$ for all $y \in A \backslash X$.

To prove the furthermore part note that $\left(\left.\left.R\right|_{(A \backslash X)} \gg I\right|_{\left\{x_{1}\right\}} \gg \ldots \gg\right.$ $\left.\left.\left.\left.\left.I\right|_{\left\{x_{k}\right\}} \backslash R^{\prime}\right|_{(A \backslash X)} \gg I\right|_{\left\{x_{1}\right\}} \gg \ldots \gg I\right|_{\left\{x_{k}\right\}}\right) \subseteq\left(R \backslash R^{\prime}\right)$. Now $\left.\left.R\right|_{(A \backslash X)} \gg I\right|_{\left\{x_{1}\right\}} \gg$ $\left.\ldots \gg I\right|_{\left\{x_{k}\right\}} \notin \mathbb{D}$ and $\left.\left.\left.R^{\prime}\right|_{(A \backslash X)} \gg I\right|_{\left\{x_{1}\right\}} \gg \ldots \gg I\right|_{\left\{x_{k}\right\}} \in \mathbb{D}$ imply $\left.R\right|_{(A \backslash X)} \gg$ $\left.\left.I\right|_{\left\{x_{1}\right\}} \gg \ldots \gg I\right|_{\left\{x_{k}\right\}} \neq\left.\left.\left. R^{\prime}\right|_{(A \backslash X)} \gg I\right|_{\left\{x_{1}\right\}} \gg \ldots \gg I\right|_{\left\{x_{k}\right\}}$. Consequently $0<\#\left(\left.\left.\left.\left.\left.\left.R\right|_{(A \backslash X)} \gg I\right|_{\left\{x_{1}\right\}} \gg \ldots \gg I\right|_{\left\{x_{k}\right\}} \backslash R^{\prime}\right|_{(A \backslash X)} \gg I\right|_{\left\{x_{1}\right\}} \gg \ldots \gg I\right|_{\left\{x_{k}\right\}}\right) \leq$ $\#\left(R \backslash R^{\prime}\right)=1$ and $\#\left(\left.\left.\left.\left.\left.R\right|_{(A \backslash X)} \gg I\right|_{\left\{x_{1}\right\}} \gg \ldots \gg I\right|_{\left\{x_{k}\right\}} \backslash R^{\prime}\right|_{(A \backslash X)} \gg I\right|_{\left\{x_{1}\right\}} \gg\right.$ $\left.\left.\ldots \gg I\right|_{\left\{x_{k}\right\}}\right)=1$.

Theorem 1 Let $\mathbb{D} \varsubsetneqq \mathbb{T}$ be a domain of preferences. Let $\varphi$ be a preference rule from $\mathbb{D}^{N}$ to $\mathbb{T}$ that is neutral, Pareto optimal and independent of irrelevant alternatives. Let $\varphi$ have no single winning agents. Then $\varphi\left(\mathbb{D}^{N}\right)$ is more structure diverse than $\mathbb{D}$.

Proof. As $\mathbb{D}$ is a proper subset of the set of tournaments $\mathbb{T}$ there exist $R \in \mathbb{T}$ with $R \notin \mathbb{D}$. Next consider $R^{\prime}$ in $\mathbb{D}$ for which $\#\left(R \backslash R^{\prime}\right)$ is minimal. There is a (swap) path of tournaments $R=R^{0}, R^{1}, \ldots, R^{k}=R^{\prime}$ such that $R^{t} \backslash R^{t+1}=$ $\left\{\left(x^{t}, y^{t}\right)\right\}$ for $t \in\{0,1, \ldots, k-1\}$ and $\left\{\left(x^{t}, y^{t}\right): t \in\{0,1, \ldots, k-1\}\right\}=R \backslash R^{\prime}$. Clearly $R^{k-1}$ is not in $\mathbb{D}$ as $R^{\prime}$ in $\mathbb{D}$ minimizes $\#\left(R \backslash R^{\prime}\right)$. So, without loss of generality we may assume that $R=R^{k-1}$ and therewith that $\#\left(R \backslash R^{\prime}\right)=1$. By Lemma 2 we may assume that

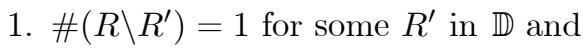

2. $R=\left.\left.\left.R\right|_{(A \backslash X)} \gg I\right|_{\left\{x_{1}\right\}} \gg \ldots \gg I\right|_{\left\{x_{k}\right\}}$ and $\left.\left.R\right|_{(A \backslash(X \cup\{y\}))} \gg I\right|_{\left\{x_{1}\right\}} \gg \ldots \gg$ $\left.\left.I\right|_{\left\{x_{k}\right\}} \gg I\right|_{\{y\}}$ is in $\mathbb{D}$ for all $y \in A \backslash X$ where $X=\left\{x_{1}, x_{2}, \ldots, x_{k}\right\}$ is a subset of $A$.

Next we show that $R$ is in $\varphi\left(\mathbb{D}^{N}\right)$ which in view of Proposition 2 is sufficient to complete the proof. As $\#\left(R \backslash R^{\prime}\right)=1$ there is precisely one pair of alternatives $(a, b)$ which is in $R$ but not in $R^{\prime}$. Let $X_{i n}=\{x \in X \backslash\{a\}:(x, a) \in R\}$ and $X_{\text {out }}=\{x \in X \backslash\{a\}:(a, x) \in R\}$. Note that $b \in X_{\text {out }}$. By Lemma 1 there are winning coalitions $S, T$ and $V$ such that $S \cap T, S \cap V$ and $T \cap V$ partition $N$. Consider profile $p$ for which we will show that $\varphi(p)=R$. This profile is defined for an agent $i \in N$ as follows

$$
\begin{aligned}
& \left.\left.\left.R\right|_{X_{\text {in }}} \gg I\right|_{\{a\}} \gg R\right|_{X_{\text {out }}}=p(i) \text { for } i \in S \cap T \\
& \left.\left.I\right|_{\{a\}} \gg R\right|_{(A \backslash\{a\})}=p(i) \text { for } i \in T \cap V \text {. }
\end{aligned}
$$

By property 2 it follows that $\left.\left.R\right|_{(A \backslash\{a\})} \gg I\right|_{\{a\}}$ is in $\mathbb{D}$. Note that $X_{i n},\{a\}$, and $X_{\text {out }}$ partition $A$. Applying cut and paste to $\left.\left.R\right|_{(A \backslash\{a\})} \gg I\right|_{\{a\}} \in \mathbb{D}$ and $B=X_{\text {in }} \cup\{a\}$, yields that $\left.\left.\left.R\right|_{X_{\text {in }}} \gg I\right|_{\{a\}} \gg R\right|_{X_{\text {out }}}=\left.\left(\left.\left.R\right|_{X_{\text {in }}} \gg I\right|_{\{a\}}\right)\right|_{B} \gg$ $\left.\left(\left.\left.R\right|_{X_{\text {out }}} \gg I\right|_{\{a\}}\right)\right|_{(A \backslash B)}=\left.\left.\left(\left.\left.R\right|_{(A \backslash\{a\})} \gg I\right|_{\{a\}}\right)\right|_{B} \gg\left(\left.\left.R\right|_{(A \backslash\{a\})} \gg I\right|_{\{a\}}\right)\right|_{(A \backslash B)}$ is in $\mathbb{D}$. Applying cut and paste to $\left.\left.R\right|_{(A \backslash\{a\})} \gg I\right|_{\{a\}}$ and $B=\{a\}$ yields $\left.I\right|_{\{a\}} \gg$ $\left.R\right|_{(A \backslash\{a\})}=\left.\left.\left(\left.\left.R\right|_{(A \backslash\{a\})} \gg I\right|_{\{a\}}\right)\right|_{B} \gg\left(\left.\left.R\right|_{(A \backslash\{a\})} \gg I\right|_{\{a\}}\right)\right|_{(A \backslash B)}$ we obtain is in $\mathbb{D}$. So, $p$ is in $\mathbb{D}^{N}$. 
Let $(x, y) \in R$ with $x \neq y$. To prove that $\varphi(p)=R$ it is sufficient to prove that $(x, y) \in \varphi(p)$. Consider the following table of decisiveness

$\begin{array}{cccc}x \backslash y & \{a\} & X_{\text {in }} & X_{\text {out }} \\ \{a\} & \times & \times & T \\ X_{\text {in }} & S & N & N \\ X_{\text {out }} & \times & V & N\end{array}$.

It indicates by which winning coalition $(x, y)$ is in $\varphi(p)$. For instance if $x=a$ and $y \in X_{\text {out }}$ winning coalition $T$, at the second row fourth column, applies to profile $p$ to yield $(x, y) \in \varphi(p)$. Note that indeed at profile $p$ in this case all agents in $N \backslash T=S \cap V$ prefer $y$ to $x=a$ at $p$. As $N \backslash T$ is not winning we denote $\mathrm{a} \times$ at the appropriate entry in this table. It is straightforward to check this table by which the result follows.

Theorem 1 shows that when individual preferences are aggregated by a neutral and Pareto optimal preference rule that is independent of irrelevant alternatives, then the range of such a rule is more structure diverse than the preferences in the domain which are aggregated, unless the rule has winning agents. This means that in the absence of winning agents such rules loose order information in the aggregating process as by allowing for more "basic structures".

\section{Remark 2}

Note that in the proof of Theorem 1 to show that $R$ is in $\varphi\left(\mathbb{D}^{N}\right)$ condition 2 may be substituted by

2* $\left.\left.R\right|_{A \backslash\{a\}} \gg I\right|_{\{a\}}$ is in $\mathbb{L}$.

So, under same premises on $\mathbb{D}$ and $\varphi$ similarly to the proof of Theorem 1 it follows that tournaments $R$ satisfying condition 1 and this condition $2^{*}$ are in the range $\varphi\left(\mathbb{D}^{N}\right)$ of $\varphi$.

\section{Remark 3 Substitution}

Let $\varphi$ be a neutral and Pareto optimal preference rule being independent of irrelevant alternatives and having no single winning agents from $\mathbb{D}^{N}$ to $\mathbb{T}$. Consider the profile $p$ of Theorem 1 . Let $\widetilde{R}$ be a tournament in the domain $\mathbb{D}$. Let $Y$ and $Z$ be a partition of $A$, where $a \in Z$. Consider the profile $\widetilde{p}$ defined by

$$
\left.R\right|_{X_{i n} \cap Y} \gg \begin{array}{rlrl}
\left.\widetilde{R}\right|_{Z} & \left.\gg R\right|_{X_{\text {out }} \cap Y} & =p(i) \text { for } i \in S \cap T \\
\left.\widetilde{R}\right|_{Z} & \left.\gg \quad R\right|_{Y}=p(i) \text { for } i \in T \cap V . \\
\left.R\right|_{Y} & \left.\gg \quad \widetilde{R}\right|_{Z}=p(i) \text { for } i \in S \cap V
\end{array}
$$

That is $\widetilde{p}$ is obtained from $p$ by substituting $\left.\widetilde{R}\right|_{Z}$ for $\left.I\right|_{\{a\}}$. Define

$$
\begin{array}{ccc}
\operatorname{Sub}\left(\left.R\right|_{Y \cup\{a\}}, a,\left.\widetilde{R}\right|_{Z}\right)=\left.R\right|_{Y} & \left.\cup \widetilde{R}\right|_{Z} & \\
\cup & \left\{(x, y) \in Y \times Z:\left.(x, a) \in R\right|_{Y \cup\{a\}}\right\} \\
& \cup & \left\{(x, y) \in Z \times Y:\left.(a, y) \in R\right|_{Y}\right\} .
\end{array}
$$


That is $\operatorname{Sub}\left(\left.R\right|_{Y \cup\{a\}}, a,\left.\widetilde{R}\right|_{Z}\right)$ is the tournament obtained from $\left.R\right|_{Y \cup\{a\}}$ by substituting $a$ with $\left.\widetilde{R}\right|_{Z}$. Like in the proof of Theorem 1 we get $\varphi(\widetilde{p})=$ $\operatorname{Sub}\left(\left.R\right|_{Y \cup\{a\}}, a,\left.\widetilde{R}\right|_{Z}\right)$, where the table of decisiveness is as follows

$\begin{array}{cccc}x \backslash y & Z & X_{\text {in }} \cap Y & X_{\text {out }} \cap Y \\ Z & N & \times & T \\ X_{\text {in }} \cap Y & S & N & N \\ X_{\text {out }} \cap Y & \times & V & N\end{array}$.

\section{Applications with respect to transitivity}

Here we show that Theorem 1 implies the classical well-known impossibility result, when in the collective preference we exclude cycles with length smaller than or equal to the number of alternatives $m$. This condition formalizes as follows.

For integer $k \geq 3$ we say that tournament $R$ is $k$-transitive if $\left(a_{1}, a_{k}\right) \in R$ for all distinct alternatives $a_{1}, a_{2}, \ldots, a_{k}$ with $\left(a_{t}, a_{t+1}\right) \in R$ for all $1 \leq t<k$. Note that the usual notion of transitivity coincides with 3-transitivity. Also note that 4-transitivity excludes cycles with length 4 but allows for cycles of length three. And so on $k$-transitivity excludes cycles with length $k$ but allows for cycles with a strictly smaller length.

It is straight forward to check that the set of all $k$-transitive tournaments is a domain of preferences.

First we deduce that if the range of a Pareto optimal and independent of irrelevant alternatives preference rule satisfies a transitivity condition the preference rule is neutral. Furthermore, in that case winning is monotone, meaning that for coalitions $S$ and $T$, with $S \subset T, T$ is winning whenever $S$ is winning.

Lemma 3 Let $\mathbb{D} \varsubsetneqq \mathbb{T}$ be a domain of preferences. Let preference rule $\varphi$ from $\mathbb{D}^{N}$ to $\mathbb{T}$ be Pareto optimal preference and independent of irrelevant alternatives. Let all tournaments in the range $\varphi\left(\mathbb{D}^{N}\right)$ be $k$-transitive, where $k \leq m$. Preference rule $\varphi$ is neutral and winning is monotone.

Proof. (Neutrality) Let $S \subseteq N$. Define $\mathcal{D}_{S}=\{(x, y):(x, y) \in \varphi(p)$ for all profiles $p$ with $(x, y) \in p(i)$ for all agents $i$ in $S$ and $(y, x) \in p(i)$ for all agents $i$ in $N \backslash S\}$. Note that $\mathcal{D}_{S}$ contains the pairs on which $S$ is winning. As $\varphi$ is independent of irrelevant alternatives $\mathcal{D}_{S}=\{(x, y):(x, y) \in \varphi(p)$ for some profiles $p$ with $(x, y) \in p(i)$ for all agents $i$ in $S$ and $(y, x) \in p(i)$ for all agents $i$ in $N \backslash S\}$. That is under the independence of irrelevant alternatives winning on $(x, y)$ at some profile with maximal opposition means winning $(x, y)$ at all profile with maximal opposition. To prove neutrality it is sufficient to show that $\mathcal{D}_{S} \neq I$ implies $A \times A=\mathcal{D}_{S}$. Therefore it is sufficient to prove for every three distinct alternatives $x, y, \geq$ and $z$, with $(x, y) \in \mathcal{D}_{S}$ and $S \subseteq T \subseteq N$ that

1. $(x, z) \in \mathcal{D}_{T}$ and

2. $(z, y) \in \mathcal{D}_{T}$. 
To prove (1) consider profile $p$ as follows

$\begin{array}{cccccccccccccc}x & y & a_{3} & a_{4} & \ldots & a_{k-1} & z & a_{k} & \ldots & a_{m} & = & p(i) & \text { for } & i \in S \\ y & x & a_{3} & a_{4} & \ldots & a_{k-1} & z & a_{k} & \ldots & a_{m} & = & p(i) & \text { for } i \in T \backslash S \\ y & a_{3} & a_{4} & \ldots & a_{k-1} & z & x & a_{k} & \ldots & a_{m} & = & p(i) & \text { for } i \in N \backslash T,\end{array}$

where $a_{3}, \ldots, a_{k-1}$ are $k-3$ distinct alternatives in $A \backslash\{x, y, z\}$. As linear orders are in all domains of preferences $p$ is in $\mathbb{D}^{N}$. As $(x, y) \in \mathcal{D}_{S}$ it follows that $(x, y) \in$ $\varphi(p)$, and Pareto optimality implies $\left(y, a_{3}\right),\left(a_{3}, a_{4}\right), \ldots,\left(a_{k-2}, a_{k-1}\right),\left(a_{k-1}, z\right)$ $\in \varphi(p)$. So, $k$-transitivity implies $(x, z) \in \varphi(p)$ and therewith $(x, z) \in \mathcal{D}_{T}$. Similarly we can prove (2).

(Monotonicity) Let $S$ be winning and $S \subset T$. It is sufficient to prove that $T$ is winning. For $S \subseteq T \subseteq N$ part (1) implies that if $A \times A=\mathcal{D}_{S} \neq I$, then $\mathcal{D}_{T} \neq I$. So, like above it follows that $\mathcal{D}_{T}=A \times A$. This means that $T$ is winning.

We say that preference rule $\varphi$ is dictatorial if there is an agent $j$ such that a coalition $S$ is winning if and only if $j \in S$. In that case $j$ is also called the dictator.

Theorem 2 Let $k \leq m$. Let $\mathbb{D}$ be the set all $k$-transitive tournaments. Let $\varphi$ be a Pareto optimal preference rule from $\mathbb{D}^{N}$ to $\mathbb{T}$, which additionally is independent of irrelevant alternatives. Let all tournaments in the range $\varphi\left(\mathbb{D}^{N}\right)$ be $k$-transitive, where $k \leq m$. Then $\varphi$ is dictatorial.

Proof. Lemma 3 implies that $\varphi$ is neutral. As $\varphi\left(\mathbb{D}^{N}\right)$ consists of $k$-transitive tournaments only, we have $\varphi\left(\mathbb{D}^{N}\right) \subseteq \mathbb{D}$. So, Theorem 1 implies that there is a winning single agent, say $j$. As by Lemma 3 winning is monotone it follows that $\varphi$ is dictatorial with dictator $j$.

Theorem 2 shows that unless a Pareto optimal and independent of irrelevant alternatives preference rule is dictatorial collective preferences in its range do not satisfy the same transitivity conditions as the individual preferences in its domain. The following Theorem shows that independent of the domain of individual preferences the collective preferences in the range of a Pareto optimal and not dictatorial preference rule which is independent of irrelevant alternatives may violate $k$-transitive for every $k \leq m$.

Theorem 3 Let $\mathbb{D}$ be domain of preferences. Let $\varphi$ be a Pareto optimal preference rule from $\mathbb{D}^{N}$ to $\mathbb{T}$ which additionally is independent of irrelevant alternatives and not dictatorial. Then for all $k \leq m$ there are tournaments in $\varphi\left(\mathbb{D}^{N}\right)$ that are not $k$-transitive.

Proof. If $\mathbb{D}=\mathbb{T}$, then as by Pareto optimality $\mathbb{D} \subseteq \varphi\left(\mathbb{D}^{N}\right)$, it follows that $\varphi\left(\mathbb{D}^{N}\right)=\mathbb{T}$. In that case the Theorem follows evidently. So, let $\mathbb{D} \varsubsetneqq \mathbb{T}$. To the contrary for this case suppose there is a number $k \leq m$ such that all collective preferences in $\varphi\left(\mathbb{D}^{N}\right)$ that are $k$-transitive. Then by Lemma 3 it follows that $\varphi$ is neutral and winning is monotone. So, there are no winning agents, as otherwise by the monotonicity of winning $\varphi$ would have been dictatorial. As all 
preferences in $\varphi\left(\mathbb{D}^{N}\right)$ that are $k$-transitive, $\widehat{T}_{k}$ is not in $\varphi\left(\mathbb{D}^{N}\right)$. But $\widehat{T}_{k}$ is just one preference swap, of $\left(a_{k}, a_{1}\right)$ to $\left(a_{1}, a_{k}\right)$, away from linear order $\widehat{R}$. As the linear orders are contained in $\mathbb{D}$ we have a contradiction because by Remark 2 it follows that $\widehat{T}_{k}$ is in $\varphi\left(\mathbb{D}^{N}\right)$.

\section{Applications with respect to structure diver- sity}

Let both domains $\mathbb{D}$ and $\mathbb{D}^{\prime}$ be closed under permutations and cut and paste. Domain $\mathbb{D}^{\prime}$ is said to be minimally more structure diverse than domain $\mathbb{D}$, notation $\mathbb{D} \subset_{m} \mathbb{D}^{\prime}$, if

1. $\mathbb{D} \varsubsetneqq \mathbb{D}^{\prime}$ and

2. $\mathbb{S}=\mathbb{D}$ or $\mathbb{S}=\mathbb{D}^{\prime}$ for all sets of tournament $\mathbb{S}$ which are closed under both permutations and cut and paste and $\mathbb{D} \subseteq \mathbb{S} \subseteq \mathbb{D}^{\prime}$.

Lemma 4 can also be found in Storcken en de Swart (19..) because the model at hand is slightly different here we state and proof the Lemma ${ }^{1}$. For a set of tournaments $\mathbb{S}$ let $\overline{\mathbb{S}}$ denote the (inclusion) smallest set that is closed under permutations and cut and paste and that contains $\mathbb{S}^{2}$.

Lemma 4 Let domain $\mathbb{D}^{\prime}$ be minimally more structure diverse than domain $\mathbb{D}$. Then there are $R \notin \mathbb{D}$ and subset $X=\left\{x_{1}, x_{2}, \ldots, x_{k}\right\}$ of $A$ such that

1. $R=\left.\left.\left.R\right|_{(A \backslash X)} \gg I\right|_{\left\{x_{1}\right\}} \gg \ldots \gg I\right|_{\left\{x_{k}\right\}}$,

2. $\left.\left.\left.\left.R\right|_{(A \backslash(X \cup\{y\}))} \gg I\right|_{\left\{x_{1}\right\}} \gg \ldots \gg I\right|_{\left\{x_{k}\right\}} \gg I\right|_{\{y\}}$ is in $\mathbb{D}$ for all $y \in A \backslash X$ and

3. $\mathbb{D}^{\prime}=\overline{\mathbb{D} \cup\{R\}}$

Moreover, let $R^{\prime} \in \mathbb{D}^{\prime}$. Let $Y=\left\{y_{1}, y_{2}, \ldots, y_{k-1}\right\}$ and $Z=\left\{z_{1}, \ldots, z_{l}\right\}$ partition A. Then $\left.\left.\left.R^{\prime}\right|_{Y} \gg I\right|_{\left\{z_{1}\right\}} \gg \ldots \gg I\right|_{\left\{z_{l}\right\}} \in \mathbb{D}$.

Proof. By Lemma 2 we may find a tournament $R$ and subset $X$ of $A$ such that (1) and (2) are satisfied. It is clear that $\mathbb{\mathbb { D }} \varsubsetneqq \overline{\mathbb{D} \cup\{R\}}$ and $\overline{\mathbb{D} \cup\{R\}} \subseteq \mathbb{D}^{\prime}$. Because $\mathbb{D}^{\prime}$ is minimally more structure diverse than domain $\mathbb{D}$ (3) follows.

For the proof of the moreover part assume that $\left.\left.R^{\prime}\right|_{Y} \gg I\right|_{\left\{z_{1}\right\}} \gg \ldots \gg$ $\left.I\right|_{\left\{z_{l}\right\}} \notin \mathbb{D}$. It is sufficient to prove that $\left.\left.\left.R^{\prime}\right|_{Y} \gg I\right|_{\left\{z_{1}\right\}} \gg \ldots \gg I\right|_{\left\{z_{l}\right\}}=\sigma R$ for some permutation $\sigma$ on $A$. Having $\left.\left.\left.R^{\prime}\right|_{Y} \gg I\right|_{\left\{z_{1}\right\}} \gg \ldots \gg I\right|_{\left\{z_{l}\right\}} \notin \mathbb{D}$, we may find a number $k^{\prime}$ subset $X^{\prime}=\left\{x_{1}^{\prime}, x_{2}^{\prime}, \ldots, x_{k^{\prime}}^{\prime}\right\}$ of $A$ such that $Z \subseteq X^{\prime}$

\footnotetext{
${ }^{1}$ Here we do not use closedness under reversion.

${ }^{2}$ Note that

1. if $\mathbb{D}^{1}$ and $\mathbb{D}^{2}$ and close under permutations then so is $\mathbb{D}^{1} \cap \mathbb{D}^{2}$,

2. if $\mathbb{D}^{1}$ and $\mathbb{D}^{2}$ and close under cut and paste then so is $\mathbb{D}^{1} \cap \mathbb{D}^{2}$.

So, smallest is well defined.
} 
1. $R^{\prime}=\left.\left.\left.R\right|_{\left(A \backslash X^{\prime}\right)} \gg I\right|_{\left\{x_{1}^{\prime}\right\}} \gg \ldots \gg I\right|_{\left\{x_{k}^{\prime}\right\}}$,

2. $\left.\left.\left.\left.R^{\prime}\right|_{(A \backslash(X \cup\{y\}))} \gg I\right|_{\left\{x_{1}^{\prime}\right\}} \gg \ldots \gg I\right|_{\left\{x_{k}^{\prime}\right\}} \gg I\right|_{\{y\}}$ is in $\mathbb{D}$ for all $y \in A \backslash X^{\prime}$ and

3. $\mathbb{D}^{\prime \prime}=\overline{\mathbb{D} \cup\left\{R^{\prime}\right\}}$.

As $R^{\prime} \in \mathbb{D}^{\prime}$ we have $\mathbb{D}^{\prime \prime} \subseteq \mathbb{D}^{\prime}$. Since $\mathbb{D}^{\prime}$ is a minimal extension of $\mathbb{D}$, it follows that $\mathbb{D}^{\prime \prime}=\mathbb{D}^{\prime}$. So, $\overline{\mathbb{D} \cup\left\{R^{\prime}\right\}}=\overline{\mathbb{D} \cup\{R\}}$. Obviously, as both $R^{\prime}, R \notin \mathbb{D}$, this means that $R^{\prime}=\sigma R$ for some permutation $\sigma$ on $A$. So, $\left.\left.R^{\prime}\right|_{Y} \gg I\right|_{\left\{z_{1}\right\}} \gg \ldots \gg$ $\left.I\right|_{\left\{z_{l}\right\}}=\sigma R$.

Let $R$ be a tournament and $B$ a non-empty subset of $A$. Restricted tournament $\left.R\right|_{B}$ is called reducible if $\left.R\right|_{B}=\left.\left.R\right|_{B_{1}} \gg R\right|_{B_{2}}$ for some partition $B_{1}$ and $B_{2}$ of $B$. If $\left.R\right|_{B}$ is not reducible it is called irreducible. Note that this means that $\left.R\right|_{B}$ contains a Hamilton circuit. Let $\mathbb{D} \subset_{m} \mathbb{D}^{\prime}$. Taking $R$ and $X$ like in Lemma 4 note that for all $\emptyset \neq Y \varsubsetneqq A \backslash X$ we have that $\left.R\right|_{(A \backslash X)} \neq\left.\left. R\right|_{Y} \gg R\right|_{(A \backslash(X \cup Y))}$. As otherwise by cut and paste closedness $\left.\left.R\right|_{Y} \gg L\right|_{(A \backslash Y)}$, and $\left.L\right|_{(X \cup Y)} \gg$ $\left.R\right|_{(A \backslash(X \cup Y))}$ are in $\mathbb{D}$ for some linear order $L$ in $\mathbb{D}$. This then would imply with cut and paste closedness that $R=\left.\left.\left.\left.R\right|_{Y} \gg R\right|_{(A \backslash(X \cup Y))} \gg I\right|_{\left\{x_{1}\right\}} \gg \ldots \gg I\right|_{\left\{x_{k}\right\}}$ is in $\mathbb{D}$. This contradicts $R \notin \mathbb{D}$. So, then $\left.R\right|_{(A \backslash X)}$ is irreducible.

Theorem 4 Let $\mathbb{D}$ be a domain. Let $\varphi$ be a neutral, and Pareto optimal preference rule which additionally is independent of irrelevant alternatives from $\mathbb{D}^{N}$ to $\mathbb{T}$ and which has no single winning agent. Let $R \in \mathbb{T} \backslash \mathbb{D}$ be irreducible and $X=\left\{x_{1}, x_{2}, \ldots, x_{k}\right\}$ be a subset of $A$ such that $R=\left.\left.R\right|_{(A \backslash X)} \gg I\right|_{\left\{x_{1}\right\}} \gg \ldots \gg$ $\left.I\right|_{\left\{x_{k}\right\}}$. Let $\mathbb{D} \subset_{m} \mathbb{D}_{1} \subset_{m} \mathbb{D}_{2} \subset_{m} \mathbb{D}_{3} \subset_{m} \ldots \subset_{m} \mathbb{D}_{k}$. Then $\varphi\left(\mathbb{D}^{N}\right) \nsubseteq \mathbb{D}_{k}$.

Proof. Without loss of generality we may assume that $\left.\left.R\right|_{(A \backslash(X \cup\{y\}))} \gg I\right|_{\left\{x_{1}\right\}} \gg$ $\left.\left.\ldots \gg I\right|_{\left\{x_{k}\right\}} \gg I\right|_{\{y\}}$ is in $\mathbb{D}$ for all $y \in A \backslash X$. Further, without loss of generality we may assume that $\delta\left(\left.\left.\left.R^{\prime}\right|_{B} \gg I\right|_{\left\{x_{1}\right\}} \gg \ldots \gg I\right|_{\left\{x_{k}\right\}},\left.\left.R\right|_{B} \gg I\right|_{\left\{x_{1}\right\}} \gg \ldots \gg\right.$ $\left.\left.I\right|_{\left\{x_{k}\right\}}\right)=1$ for some $\left.\left.\left.R^{\prime}\right|_{B} \gg I\right|_{\left\{x_{1}\right\}} \gg \ldots \gg I\right|_{\left\{x_{k}\right\}} \in \mathbb{D}$. So, for some pair $(a, b)$ in $\left.\left.\left.R\right|_{B} \gg I\right|_{\left\{x_{1}\right\}} \gg \ldots \gg I\right|_{\left\{x_{k}\right\}}$ the pair $(b, a)$ is in $\left.\left.R^{\prime}\right|_{B} \gg I\right|_{\left\{x_{1}\right\}} \gg$ $\left.\ldots \gg I\right|_{\left\{x_{k}\right\}}$. Now by Remark $3 \operatorname{Sub}\left(\left.R\right|_{B \backslash\{a\}}, a,\left.\left.\left.I\right|_{\{a\}} \gg I\right|_{\left\{x_{1}\right\}} \gg \ldots \gg I\right|_{\left\{x_{k}\right\}}\right)$ is in $\varphi\left(\mathbb{D}^{N}\right)$. For $t \in\{0,1,2, \ldots, k\}$ let $\widetilde{R}^{t}=\operatorname{Sub}\left(\left.R\right|_{B \backslash\{a\}}, a,\left.\left.I\right|_{\{a\}} \gg I\right|_{\left\{x_{1}\right\}} \gg\right.$ $\left.\left.\ldots \gg I\right|_{\left\{x_{k}\right\}}\right)\left.\left.\left.\right|_{\left(A \backslash\left\{x_{t}, x_{t+1}, \ldots, x_{k}\right\}\right.} \gg I\right|_{\left\{x_{t}\right\}} \gg \ldots \gg I\right|_{\left\{x_{k}\right\}}$, where $x_{0}=a$. Let $\widetilde{\mathbb{D}}_{0}=\overline{\mathbb{D} \cup\left\{\widetilde{R}^{0}\right\}}$ and $\widetilde{\mathbb{D}}_{t+1}=\overline{\mathbb{D} \cup\left\{\widetilde{R}^{t}\right\}}$ for $1 \leq t \leq k$. Clearly $\widetilde{R}^{k} \in \varphi\left(\mathbb{D}^{N}\right)$. Next we prove that $\widetilde{R}^{t} \notin \widetilde{\mathbb{D}}_{t-1}$ for all for $1 \leq t \leq k$. If $\widetilde{R}^{t} \in \widetilde{\mathbb{D}}_{t-1}$, then by the moreover part of Lemma 4 we have the contradiction $\widetilde{R}^{t-1}=\left.\widetilde{R}^{t}\right|_{\left(A \backslash\left\{x_{t-1}, x_{t}, x_{t+1}, \ldots, x_{k}\right\}\right.} \gg$ $\left.\left.I\right|_{\left\{x_{t-1}\right\}} \gg \ldots \gg I\right|_{\left\{x_{k}\right\}} \in \widetilde{\mathbb{D}}_{t-1}$. This means that it needs minimally $k+1$ minimal extension from $\mathbb{D}$ to reach a domain that contains $\widetilde{R}^{k}$. 


\section{References}

1. Anand P.,1993, The philosophy of intransitive preferences, The Economic Journal, 103, p. 337-346.

2. Arrow K.J.,1978, Social choice and individual values, Yale University Press (19 ${ }^{\text {th }}$ edition).

3. Tversky, A., 1969, Intransitivity of preferences, Psychological Review, 76, p. 31-48.

4. de Swart, H., Storcken, T., 1992, Towards an axiomatization of orderings, Order, 9, p. 367-396. 